\title{
Shifting nodal-plane suppressions in high-order-harmonic spectra from diatomic molecules in orthogonally polarized driving fields
}

\author{
T. Das and C. Figueira de Morisson Faria \\ Department of Physics and Astronomy, University College London, Gower Street, London WC1E 6BT, United Kingdom
}

(Received 12 February 2016; published 10 August 2016)

\begin{abstract}
We analyze the imprint of nodal planes in high-order-harmonic spectra from aligned diatomic molecules in intense laser fields whose components exhibit orthogonal polarizations. We show that the typical suppression in the spectra associated to nodal planes is distorted, and that this distortion can be employed to map the electron's angle of return to its parent ion. This investigation is performed semianalytically at the single-molecule response and single-active orbital level, using the strong-field approximation and the steepest descent method. We show that the velocity form of the dipole operator is superior to the length form in providing information about this distortion. However, both forms introduce artifacts that are absent in the actual momentum-space wave function. Furthermore, elliptically polarized fields lead to larger distortions in comparison to two-color orthogonally polarized fields. These features are investigated in detail for $\mathrm{O}_{2}$, whose highest occupied molecular orbital provides two orthogonal nodal planes.
\end{abstract}

DOI: 10.1103/PhysRevA.94.023406

\section{INTRODUCTION}

Strong, orthogonally polarized fields have a wide range of applications in the attosecond imaging of molecules, and provide access to a parameter range difficult to be probed by other means. This includes molecules that are difficult to align, degenerate orbitals, or the reconstruction of molecular orbitals from a single-shot measurement, which have been probed using high-order-harmonic generation (HHG) [1-7]. Thereby, one makes use of the fact that HHG is the result of the recollision of a previously ionized electron with its parent ion [8]. Orthogonally polarized fields provide a means of controlling the angle with which the active electron reaches the continuum, or returns to the molecular target $[2,4,5]$. This angle varies with the harmonic frequency [9]. Recently, two-color fields with orthogonal polarizations have also been used to control resonancelike enhancements near channel closings [10]. This control is made possible by the fact that, from a semiclassical viewpoint, such enhancements are caused by the quantum interference of many electron orbits returning to the target, whose features are influenced by the field [11].

Care must be taken, however, as the orthogonal polarization introduces dynamical aspects in purely structural features. A good example is the interference patterns related to electron recollision in different centers in the molecule. For linearly polarized fields, these patterns have been widely studied within the single active electron, single active orbital approximation. They depend only on the internuclear distance, the geometry of the active orbital, and on the molecule orientation with regard to the field [12,13] (for reviews see, e.g., [14] and our recent publication [15]). This is in striking contrast to what happens if the field is orthogonally polarized. In fact, in a recent publication we have shown that the electron's angle of return is effectively incorporated in the two-center interference condition [16]. Because this angle depends on the harmonic energy and the orbit along which the electron returns, a coherent superposition of orbits will lead to a blurring in the structural minima. This blurring has also been identified, but not analyzed, in $[17,18]$. Fortunately, the contribution of specific orbits can be eliminated by appropriately chosen macroscopic propagation conditions. This means that the shift caused by a nonvanishing angle of return can be observed in a realistic scenario [19].

In this paper, we assess the influence of orthogonal fields in the suppression that occurs across the HHG spectrum when a molecular nodal plane is in alignment with the driving-field polarization (see, e.g., [20-23]). This suppression arises from the fact that nodal planes are areas of vanishing probability density. Hence, either ionization is strongly suppressed or the overlap between the recolliding electronic wave packet and the core wave function is vanishingly small [24-26]. One should note that this specific feature is related to the geometry of the orbital with which the active electron recombines, and not to the structural interference studied in $[16,19]$. Thus, nodal planes provide an additional tool to those discussed in our previous work [16]. As a target, we employ $\mathrm{O}_{2}$, whose highest occupied molecular orbital (HOMO) exhibits two orthogonal nodal planes. We also investigate which type of driving fields, of one or two colors, is the most appropriate for observing such distortions. The present studies are different in the sense that distorted nodal-plane suppressions in elliptically polarized fields can be seen in [17], but they have not been analyzed. Furthermore, previous publications that have addressed nodalplane suppressions and elliptical fields [27,28] have focused on ionization, but not on recombination. They found that, although, on their own, nodal planes and elliptical fields suppress ionization, the combination of both can in fact compensate for each other. This increases the HHG signal when the major polarization axis and the nodal plane are in alignment.

Throughout, we use the strong-field approximation (SFA) and the steepest descent method. This approach has been widely employed in the theoretical study of molecular HHG [17,20,21,29-35], as (i) it allows an intuitive interpretation in terms of electron orbits, and (ii) by being analytic, the SFA provides a high degree of flexibility, as the influence of its building blocks in the HHG spectra can be singled out at will. The SFA has also been successfully used to infer an electron's angle of return in orthogonally polarized fields from the ratio between even and odd harmonics in experiments [36]. Furthermore, in [4], it has been shown, in the context of 
strong-field ionization, that the returning angle predicted by the SFA corresponds to the average angle obtained from a classical-trajectory calculation. The SFA, however, has serious limitations. Apart from the gauge dependence and its influence on the structural interference [32,33,37], different forms of the recombination dipole matrix element affect the HHG spectra $[21,38,39]$. The most appropriate form to be used has raised considerable debate in the context of the tomographical reconstruction of molecular orbitals [40-42]. It is generally accepted that the velocity form is superior when dealing with molecules, but that the artifacts introduced by the length form are under control and can be eliminated. In this work, we revisit this issue and show that orthogonally polarized fields expose artifacts in the length form of the SFA, which cannot be probed with linearly polarized fields.

This article is organized as follows. In Sec. II, we provide the necessary theoretical background. In Sec. III, we compute HHG spectra using one- and two-color orthogonally polarized fields, and analyze the features encountered. This includes the nodal-plane distortions for individual orbits, the most convenient form of the strong-field approximation, and the most favorable field configurations in order to observe the shifts. Finally, in Sec. IV, we state our conclusions.

\section{MODEL}

Below, we explicitly give the orthogonally polarized fields employed in this work. The electric field and the corresponding vector potential read

$$
\mathbf{E}(t)=E_{\|}(t) \hat{\epsilon}_{\|}+E_{\perp}(t) \hat{\epsilon}_{\perp}
$$

and

$$
\mathbf{A}(t)=A_{\|}(t) \hat{\epsilon}_{\|}+A_{\perp}(t) \hat{\epsilon}_{\perp}
$$

respectively, where the subscripts $(\|)$ and $(\perp)$ designate field components parallel to the major and minor polarization axes, respectively. The unit vectors along the major and the minor polarization axes are denoted by $\hat{\epsilon}_{\|}$and $\hat{\epsilon}_{\perp}$, respectively. Both fields are related to each other through $\mathbf{E}(t)=-d \mathbf{A}(t) / d t$.

\section{A. Transition amplitude}

For orthogonally polarized driving fields, the HHG spectrum is proportional to

$$
S(\Omega) \propto \sum_{b}\left|M_{b}(\Omega)\right|^{2}
$$

where $b=\|, \perp$ refer to the components of the HHG transition amplitude $\mathbf{M}(\Omega)$ parallel and perpendicular to the major polarization axis of the laser field [17,18]. We compute the transition probability $\left|M_{\|}(\Omega)\right|^{2}$, which gives the polarization component of the harmonics along the major axis. This contribution is much larger than that from the perpendicular component for the ellipticity ranges used, and provides the main features in the spectra.

The transition amplitude is computed using the SFA [43], which has been extended to orthogonally polarized fields [16].
Explicitly,

$$
\begin{aligned}
M_{\|}(\Omega)= & -i \int_{-\infty}^{\infty} d t \int_{-\infty}^{t} d t^{\prime} \int d^{3} \mathbf{p d}_{\mathrm{rec}}^{*}(\mathbf{p}+\mathbf{A}(t)) \\
& \times \mathbf{d}_{\mathrm{ion}}\left(\mathbf{p}+\mathbf{A}\left(t^{\prime}\right)\right) e^{i S\left(t, t^{\prime}, \Omega, \mathbf{p}\right)}+\text { c.c. }
\end{aligned}
$$

For the field in Eqs. (1) and (2), the semiclassical action is given by

$$
\begin{aligned}
S\left(t, t^{\prime}, \Omega, \mathbf{p}\right)= & -\frac{1}{2} \int_{t^{\prime}}^{t} d \tau\left[p_{\|}+A_{\|}(\tau)\right]^{2} \\
& -\frac{1}{2} \int_{t^{\prime}}^{t} d \tau\left[p_{\perp}+A_{\perp}(\tau)\right]^{2}-I_{p}\left(t-t^{\prime}\right)+\Omega t .
\end{aligned}
$$

Equation (5) describes the propagation of an electron in the continuum with intermediate momentum $\mathbf{p}$ from the time in which it ionizes, $t^{\prime}$, to the time $t$ when it recombines to its parent molecule. In the above-stated equations, $I_{p}$ and $\Omega$ give the ionization potential, and the harmonic frequency, respectively. The indices $b=\|$ and $b=\perp$ refer to the momentum components along the major or minor polarization axis, respectively.

Equation (4) is calculated using the steepest descent method, in which we find $t^{\prime}, t$, and $\mathbf{p}$ for which the action is stationary. The resulting saddle-point equations give the conservation of energy upon tunnel ionization and recombination, and introduce constraints in the electron momentum so that it returns to its parent molecule. It is important to bear in mind that, since tunneling is classically forbidden, the solutions of these equations will be complex. For details we refer to [16].

We employ a specific uniform approximation, which treats pairs of orbits collectively. When computing the transition probabilities associated with individual orbits we use the standard saddle-point approximation, which treats the orbits individually. This method can break down for one of the orbits when the imaginary part of the solutions diverges, leading to an increase in the harmonic yield after the cutoff (for details see Ref. [44]).

All the information about the structure of the molecule are contained within the ionization and recombination prefactors, which, along the major polarization axis, are given by

$$
d_{\text {ion }}(\mathbf{p})=\left\langle\mathbf{p}\left|H_{I}\left(t^{\prime}\right)\right| \Psi_{0}\right\rangle
$$

and

$$
d_{\mathrm{rec}}(\mathbf{p}+\mathbf{A}(t))=\left\langle\mathbf{p}+\mathbf{A}(t)\left|\hat{\mathbf{d}} \cdot \hat{\epsilon}_{\|}\right| \Psi_{0}\right\rangle,
$$

respectively, where $\hat{\mathbf{d}}$ gives the dipole operator. We neglect the motion of the nuclei and use the single active orbital approximation, which assumes that only the HOMO contributes to HHG. For a theoretical approach which includes the core dynamics, see $[23,45]$.

We represent the HOMO wave function $\Psi_{0}(\mathbf{r})$ by a linear combination of atomic orbitals (LCAO), which gives

$$
\Psi_{0}(\mathbf{r})=\sum_{a} c_{a}\left[\psi_{a}\left(\mathbf{r}+\frac{\mathbf{R}}{2}\right)+(-1)^{\ell_{a}-m_{a}+\lambda_{a}} \psi_{a}\left(\mathbf{r}-\frac{\mathbf{R}}{2}\right)\right],
$$

where $\psi_{a}(\mathbf{r}), \mathbf{R}$, and $c_{a}$ are the atomic orbitals, the internuclear distance, and the LCAO coefficients, respectively, while $\ell_{a}$ 
and $m_{a}$ refer to the orbital and to the magnetic quantum number, respectively. The indices $\lambda_{a}=m_{a}$ correspond to gerade $(\mathrm{g})$ and $\lambda_{a}=m_{a}+1$ to ungerade $(\mathrm{u})$ orbital symmetry. The orbitals used in this work have been computed with GAMESS-UK [46].

\section{B. Different dipole forms}

The recombination prefactor (7) can be written in different forms, which will lead to different results. The form of the dipole operator should not be confused with the gauge $[21,33,39]$, which determines how the Hamiltonian is written. Explicitly, the length, velocity, and acceleration forms of the dipole operator read $\hat{\mathbf{d}}^{(l)}=\hat{\mathbf{r}}, \hat{\mathbf{d}}^{(v)}=\hat{\mathbf{p}}$, and $\hat{\mathbf{d}}^{(a)}=$ $-\nabla V(\hat{\mathbf{r}})$, respectively, where the hats denote operators. In this work we have used the length gauge, so that the interaction Hamiltonian in Eq. (6) is given by $H_{I}\left(t^{\prime}\right)=\hat{\mathbf{r}} \cdot \mathbf{E}_{\|}\left(t^{\prime}\right)$ and we consider the length and velocity forms of the dipole operator.

Thus, one can write the dipole matrix element $d_{\text {rec }}(\mathbf{p}+$ $\mathbf{A}(t))$ for the wave function (8) in the length form as

$$
\begin{aligned}
d_{\mathrm{rec}}^{(l)}(\mathbf{p}(t))= & \sum_{a} c_{a}\left[e^{i \mathbf{p}(t) \cdot \frac{\mathbf{R}}{2}}+(-1)^{\ell_{a}-m_{a}+\lambda_{a}} e^{-i \mathbf{p}(t) \cdot \frac{\mathbf{R}}{2}}\right] \\
& \times i \partial_{p_{\|}(t)} \psi_{a}(\mathbf{p}(t)),
\end{aligned}
$$

and in the velocity form as

$$
\begin{aligned}
d_{\mathrm{rec}}^{(v)}(\mathbf{p}(t))= & \sum_{a} c_{a}\left[e^{i \mathbf{p}(t) \cdot \frac{\mathbf{R}}{2}}+(-1)^{\ell_{a}-m_{a}+\lambda_{a}} e^{-i \mathbf{p}(t) \cdot \frac{\mathbf{R}}{2}}\right] \\
& \times p_{\|}(t) \psi_{a}(\mathbf{p}(t))
\end{aligned}
$$

where $\mathbf{p}(t)=\mathbf{p}+\mathbf{A}(t)$ and $\psi_{a}(\mathbf{p}(t))$ is the Fourier transform of Eq. (8). In Eq. (9), the term related to the lack of orthogonality between the bound state and continuum states that occurs in the SFA has been removed by hand. This is a widely used procedure, and it is related to the fact that this term is an artifact that blurs the two-center interference condition (see [32,33,37] for discussions).

The reference frame of the molecule is rotated by the alignment angle $\theta_{L}$ with regard to the major polarization axis of the field. If we consider $x z$ as the polarization plane, we may relate the $p_{\|}, p_{\perp}$ components to the components $p_{x}, p_{z}$ parallel and perpendicular to the molecular axis via

$$
\left(\begin{array}{c}
p_{\|} \\
p_{\perp}
\end{array}\right)=\left(\begin{array}{cc}
\cos \theta_{L} & \sin \theta_{L} \\
-\sin \theta_{L} & \cos \theta_{L}
\end{array}\right)\left(\begin{array}{c}
p_{x} \\
p_{z}
\end{array}\right) .
$$

Throughout, Gaussian-type orbitals have been used in the construction of the HOMO, and only $s$ and $p$ orbitals are included in the basis sets. In this specific case, the derivative of the momentum-space wave function in the direction of the main polarization axis reads

$$
\begin{aligned}
\partial_{p \|} \psi_{a}(\mathbf{p})= & \left(-\frac{i}{2}\right)^{\ell_{a}} b_{a} c_{a} \pi^{\frac{3}{2}} \chi_{a}^{-\ell_{a}-\frac{3}{2}} e^{-\left(p_{\|}^{2}+p_{\perp}^{2}\right) /\left(4 \chi_{a}\right)} \\
& \times\left[\mathcal{K}_{1}\left(p_{\|}, p_{\perp}, \theta_{L}\right)+\mathcal{K}_{2}\left(p_{\|}, p_{\perp}, \theta_{L}\right)\right],
\end{aligned}
$$

where

$$
\mathcal{K}_{1}\left(p_{\|}, p_{\perp}, \theta_{L}\right)=-\ell_{a} \sin \theta_{L}\left(-p_{\|} \sin \theta_{L}+p_{\perp} \cos \theta_{L}\right)^{\ell_{a}-1}
$$

and

$$
\mathcal{K}_{2}\left(p_{\|}, p_{\perp}, \theta_{L}\right)=-\frac{p_{\|}}{2 \chi_{a}}\left(-p_{\|} \sin \theta_{L}+p_{\perp} \cos \theta_{L}\right)^{\ell_{a}} .
$$

In Eqs. (12)-(14), $b_{a}$ and $\chi_{a}$ give the contraction and the exponential coefficients, respectively.

\section{Angle Of return}

The effective shift derived in [16], whose real part can be associated with the electron's angle of return, reads

$$
\zeta\left(t, t^{\prime}\right)=\arctan \left[\frac{p_{\perp}+A_{\perp}(t)}{p_{\|}+A_{\|}(t)}\right],
$$

where the stationary momentum components read

$$
p_{b}=\frac{-1}{t-t^{\prime}} \int_{t^{\prime}}^{t} A_{b}(\tau) d \tau
$$

with $b=\|, \perp$. Equation (15) shows that the angle with which the electron returns will be strongly influenced by the vectorpotential components at the electron's return time along each orbit. Furthermore, $p_{\|}$and $p_{\perp}$ are functions of the return and ionization times according to Eq. (16). Hence, different orbits will have different angles of return.

Throughout, the shift in the nodal-plane suppression will be calculated using $\operatorname{Re}\left[\zeta\left(t, t^{\prime}\right)\right]$. A similar expression has been used in [4], as a comparison with the outcome of a classicaltrajectory computation. Therein, it was found that the mean values of the numerically determined angles are well described by the SFA.

\section{HIGH-HARMONIC SPECTRA}

In the following results, we use orthogonally polarized fields of the form

$$
\mathbf{E}(t)=\frac{E_{0}}{\sqrt{1+\xi^{2}}}\left[\sin (\omega t) \hat{\epsilon}_{\|}+\xi \sin (n \omega t-2 \pi \phi) \hat{\epsilon}_{\perp}\right],
$$

where the frequency ratio of $n=1$ gives an elliptically polarized field and $n=2$ corresponds to an orthogonally polarized two-color (OTC) field. In Eq. (17), the strength of the field component along the minor polarization axis relative to its component along the major axis is determined by $\xi$, and the relative phase $\phi$ controls the time delay between both waves. The field has been normalized in order to keep the time-averaged intensity $\left\langle\mathbf{E}^{2}(t)\right\rangle_{t}$ constant. This renders the total ponderomotive energy $U_{p}=\left\langle A_{\|}^{2}(t)\right\rangle_{t} / 2+\left\langle A_{\perp}^{2}(t)\right\rangle_{t} / 2$ constant for elliptical fields, and causes a decrease in $U_{p}$ with $\xi$ for OTC fields.

Throughout we employ $\mathrm{O}_{2}$ as a molecular target, which is particularly convenient since its HOMO is a $1 \pi_{g}$ orbital [see Fig. 1]. This leads to two nodal planes that are perpendicular to each other and produce suppressions in the HHG signal when the molecular axis of $\mathrm{O}_{2}$ is aligned at $\theta_{L}=0, \pi / 2, \pi$, and $3 \pi / 2$ with respect to the major polarization axis of the field. We also avoid the effects of two-center interference by an appropriate choice of driving-field intensity. We consider either a coherent superposition of the two dominant, shortest pair of orbits, or contributions from individual orbits. The two dominant orbits are well known in the literature as the "long orbit" and "short orbit" [47], and correspond to electron excursion 

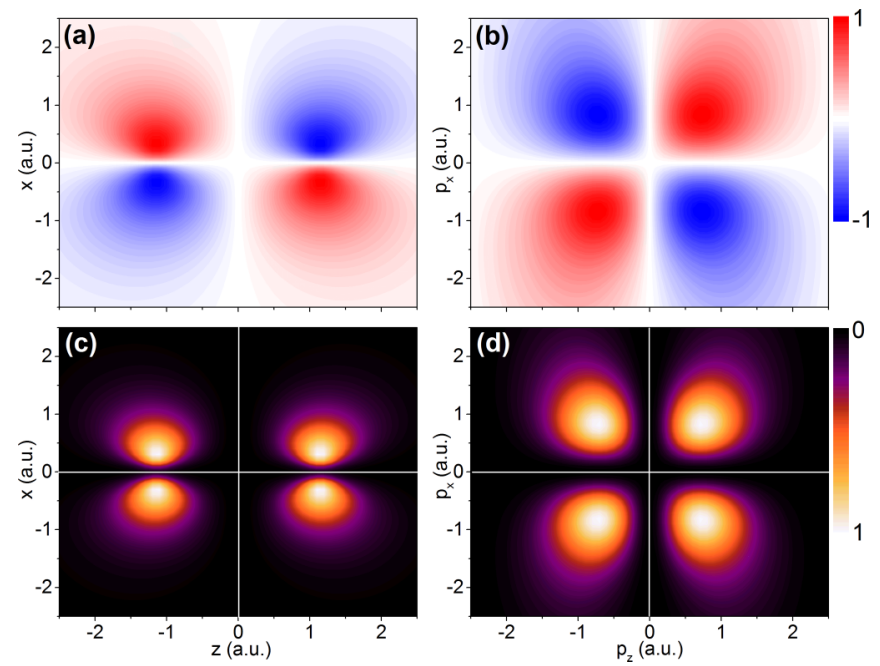

FIG. 1. Panels (a) and (b) show the real parts of the position- and momentum-space wave functions of the $\mathrm{HOMO}$ of $\mathrm{O}_{2}$, respectively, computed using Eq. (8) and its Fourier transform. Panels (c) and (d) show the corresponding probability densities $\left|\Psi_{0}(\mathbf{r})\right|^{2}$ and $\left|\Psi_{0}(\mathbf{p})\right|^{2}$, respectively. The HOMO of $\mathrm{O}_{2}$ is a $1 \pi_{g}$ orbital with ionization potential $I_{p}=12 \mathrm{eV}$ and internuclear separation $R=2.28$ a.u., with two perpendicular nodal planes. The nodal planes are designated by the white lines in the lower panels. The contours have been normalized to the maximum yield in each panel, and the blue [red] lobes correspond to the negative [positive] values of the real parts of the wave functions. In this picture, the internuclear axis is oriented along the $z$ axis. times of the order of three-quarters of a field cycle. In previous work, we have found that, for orthogonally polarized fields, the remaining orbits do not play a very important role [16]. For clarity, in the results that follow we restrict the electron ionization times to the first half cycle of the driving field.

\section{A. Individual prefactors}

The first row of Fig. 2 [Figs. 2(a) to 2(c)] shows that, for increasing driving-field ellipticity, the suppressions begin to weaken and the alignment angle for which they appear in the spectrum changes. These changes become less substantial for increasing harmonic order and seem to behave differently for suppressions around even and odd multiples of $\pi / 2$. For $\theta_{L}=$ $n \pi$, we observe more blurring and larger shifts, in comparison to the behavior near $\theta_{L}=(2 n+1) \pi / 2$.

In the remaining rows of Fig. 2, we show the effect of using only the recombination or the ionization prefactor in the calculation of $\left|M_{\|}(\Omega)\right|^{2}$, which are presented here for analytical purposes (second and third rows, respectively). These figures show us that, as the ellipticity of the field is increased, the shifted nodal-plane suppressions are determined by the recombination prefactor. All the structure in the ionization prefactor is washed out for large enough ellipticity. This is in agreement with [28], which found that, although the individual effects of the nodal plane and of the field ellipticity are detrimental to $\mathrm{HHG}$, they can cancel each other when combined.

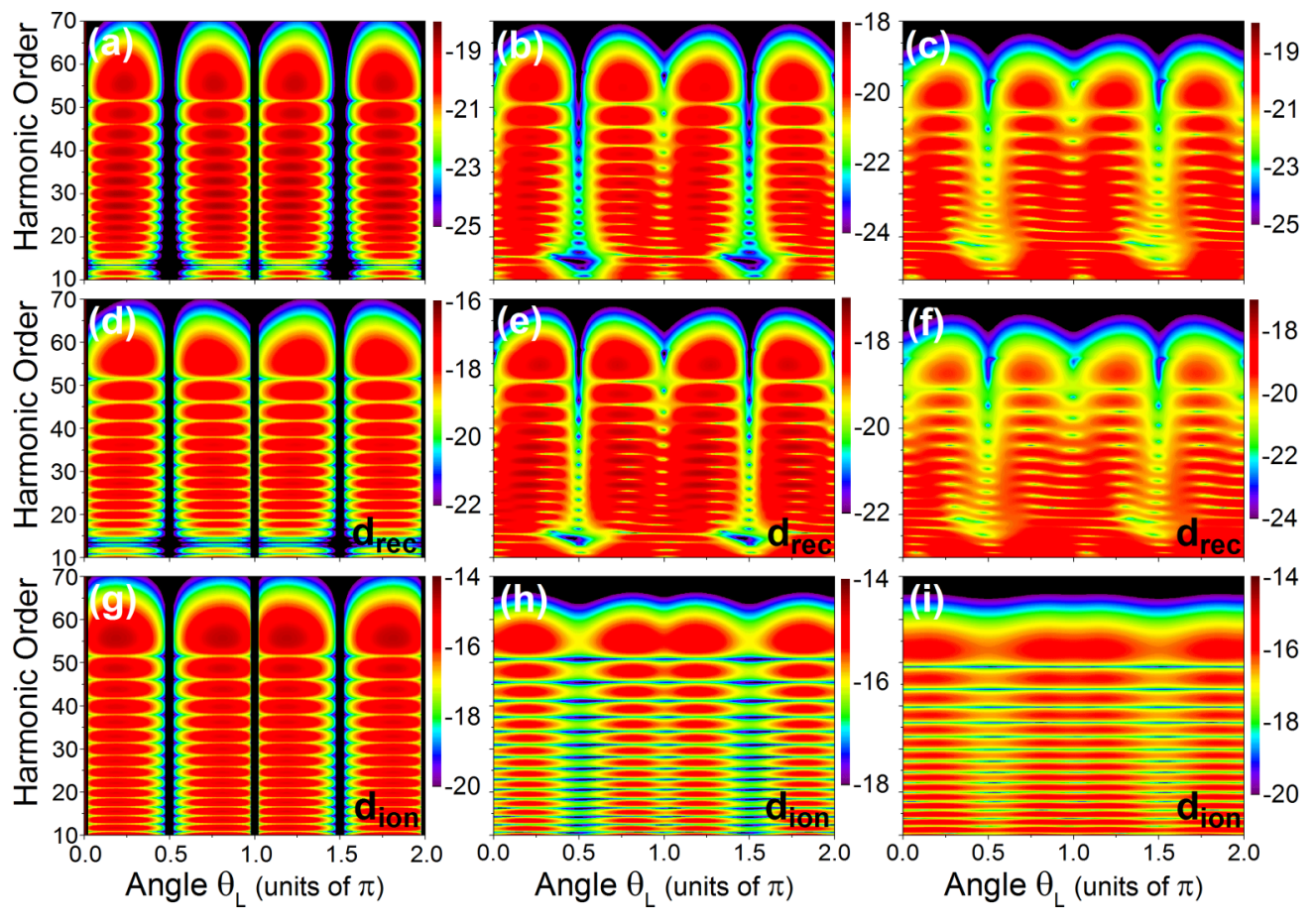

FIG. 2. Contributions $\left|M_{\|}(\Omega)\right|^{2}$ of the dipole component along the major polarization axis to the HHG spectra computed using the length form of the dipole operator for a coherent superposition of the dominant long and short orbits, as functions of the alignment angle $\theta_{L}$ for $\mathrm{O}_{2}\left(I_{p}=12 \mathrm{eV}\right.$ and internuclear separation $R=2.28$ a.u. $)$ in an elliptical field described in Eq. (17) with $n=1, \omega=0.057$ a.u., $I=4 \times 10^{14} \mathrm{Wcm}^{-2}$, and time delay $\phi=0.25$. The complete prefactor is calculated in the first row while only the recombination and ionization prefactors are used to calculate the spectra in the second and third rows, respectively. The first, second, and third columns give an increasing value of the field ellipticity of $\xi=0,0.15$, and 0.3 , respectively. All panels are displayed in a logarithmic scale. 

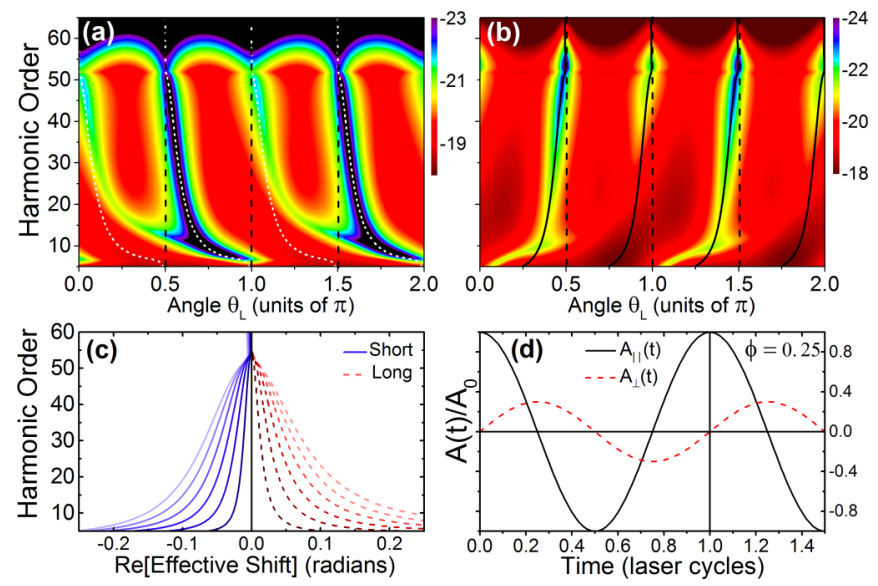

FIG. 3. Panels (a) and (b) show the transition probabilities $\left|M_{\|}(\Omega)\right|^{2}$ along the major polarization axis plotted in a logarithmic scale as functions of the alignment angle $\theta_{L}$ for $\mathrm{O}_{2}$ in an elliptical field described in Eq. (17), using the same parameters as in Fig. 2 and the length form of the dipole operator. Panel (a) [panel (b)] shows the individual contributions from the long [short] orbit. In panel (a), the shifted positions of the nodal-plane suppressions calculated using $\operatorname{Re}\left[\zeta\left(t, t^{\prime}\right)\right]$ [Eq. (15)] are indicated by the white short dashed curves, and in panel (b) they are given by the solid black lines. For comparison, we also indicate the position of the nodal-plane suppressions for linearly polarized fields as the dashed black lines. The increase in the harmonic yields after the cutoff observed in panel (b) is related to a breakdown of the standard saddle-point approximation for the short orbit (for details, see Ref. [44]). In panel (c) we have plotted the real parts of the effective shifts $\zeta\left(t, t^{\prime}\right)$ as functions of the harmonic order computed for the long (red dashed curves) and short (blue solid curves) orbits in laser fields of increasing ellipticity and the same relative phase, intensity, and frequency as in panels (a) and (b). The ellipticity has been increased from $\xi=0$ to $\xi=0.3$ in increments of $\delta \xi=0.05$. A lighter color indicates a higher ellipticity and a vanishing shift is indicated by a vertical black line. Panel (d) shows a schematic representation of the major and minor components of the vector potential $\mathbf{A}(\mathrm{t})$ for ellipticity $\xi=0.3$ and relative phase $\phi=0.25$. The electron return time at $t=2 \pi / \omega$ is indicated by the thick vertical black line in the figure. For simplicity, all fields have been normalized to the vector potential amplitude $\mathrm{A}_{0}=\mathrm{E}_{0} / \omega$.

\section{B. Individual orbits and different SFA forms}

In the upper row of Fig. 3, we show the transition probabilities $\left|M_{\|}(\Omega)\right|^{2}$ associated with the long and short orbits as functions of the alignment angle $\theta_{L}$ [panels (a) and (b), respectively], plotted in a logarithmic scale. In general, their positions are orbit dependent. For the long orbit, they are displaced to the right, while for the short orbit this displacement is to the left. We also see that the displacement decreases for both orbits with increasingly higher harmonics. At the cutoff, the shifts vanish and the suppressions occur at $\theta_{L}=(2 n+1) \pi / 2$, as in the linearly polarized case.

Figure 3(c), in which the real parts of the effective shifts $\zeta\left(t, t^{\prime}\right)$ are plotted for driving fields of increasing ellipticity, confirms this trend. It shows two approximately symmetric shifts which decrease with harmonic order until they eventually vanish at the cutoff. This happens because, around the cutoff, the electron returns at a crossing of the electric field, i.e.,

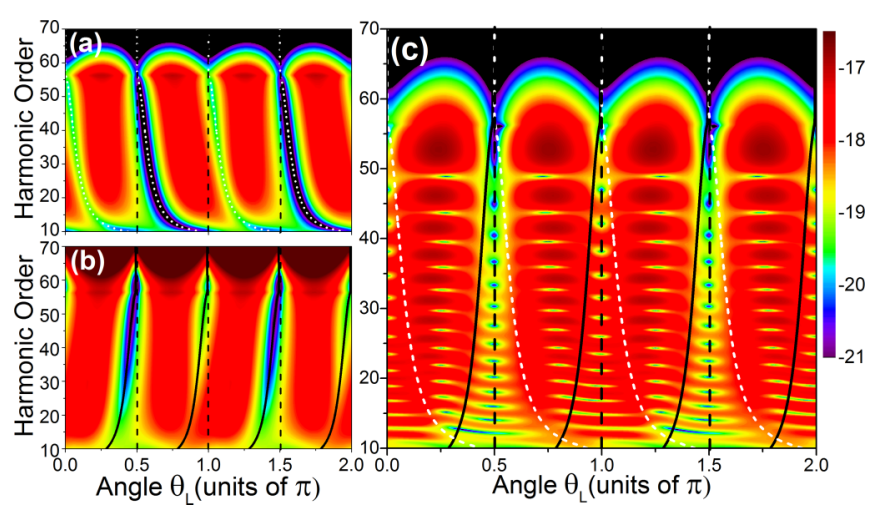

FIG. 4. Panels (a) and (b) show the transition probabilities $\left|M_{\|}(\Omega)\right|^{2}$, plotted in a logarithmic scale, for the long and short individual orbits, respectively, as functions of the alignment angle $\theta_{L}$ for $\mathrm{O}_{2}$ in an elliptical field described in Eq. (17) using the same parameters as in Fig. 2, but calculated using the velocity form of the dipole matrix elements. Panel (c) shows $\left|M_{\|}(\Omega)\right|^{2}$ for a coherent superposition of the dominant long and short orbits considered in panels (a) and (b). The shifted positions of the nodal-plane suppressions calculated using $\operatorname{Re}\left[\zeta\left(t, t^{\prime}\right)\right]$ [Eq. (15)] are indicated by the white short dashed curves for the long orbit, and by the solid black lines for the short orbit. For comparison, we also indicate the positions of the nodal-plane suppressions for linearly polarized fields as the dashed black lines. The color map on the right-hand side refers to all panels in the figure.

at a crest of $A_{\|}(t)$ [see Fig. 3(d)]. At such times, $A_{\perp}(t)=$ 0 for both orbits, which translates into a vanishing shift. Below the cutoff, the short and long orbits are subjected to equal but opposite perpendicular momenta, which increase for decreasing harmonic frequency. Hence, the nodal-plane suppressions are increasingly displaced in opposite directions for each orbit as the harmonic order decreases. Similar effects are described in more detail in Ref. [16], albeit in a different context, namely, two-center interference. This also confirms that Eq. (15) is applicable to features near nodal planes. This is not obvious, as it has been derived directly from the interference condition.

Throughout, we observe an excellent agreement between Eq. (15) and the outcome of the SFA computations for the nodal-plane suppressions that are positioned at $\theta_{L}=(2 n+$ 1) $\pi / 2$ for linearly polarized fields. For the suppressions near even multiples of $\theta_{L}=\pi / 2$, the calculated effective shift does not fit the SFA outcome. The latter is strongly exaggerated for lower harmonics, and even meet the other shifted suppressions near the ionization threshold (see white short dashed lines in the picture). We also see that the suppressions are more blurred than those encountered for $\theta_{L}=(2 n+1) \pi / 2$.

If, instead, the matrix element $d_{\mathrm{rec}}^{(v)}\left(\mathbf{p} \cdot \hat{\epsilon}_{\|}\right)$in the velocity form is used, the agreement between the SFA and the analytical condition (15) improves significantly near $\theta_{L}=0, \pi$, and $2 \pi$. There is, however, some blurring, if compared with the suppressions observed near odd multiples of $\pi / 2$ for the low harmonic ranges. These results can be seen in Fig. 4 , for the individual contributions of the long and short orbit [Figs. 4(a) and 4(b), respectively], together with the results obtained using their coherent superposition [Fig. 4(c)]. 

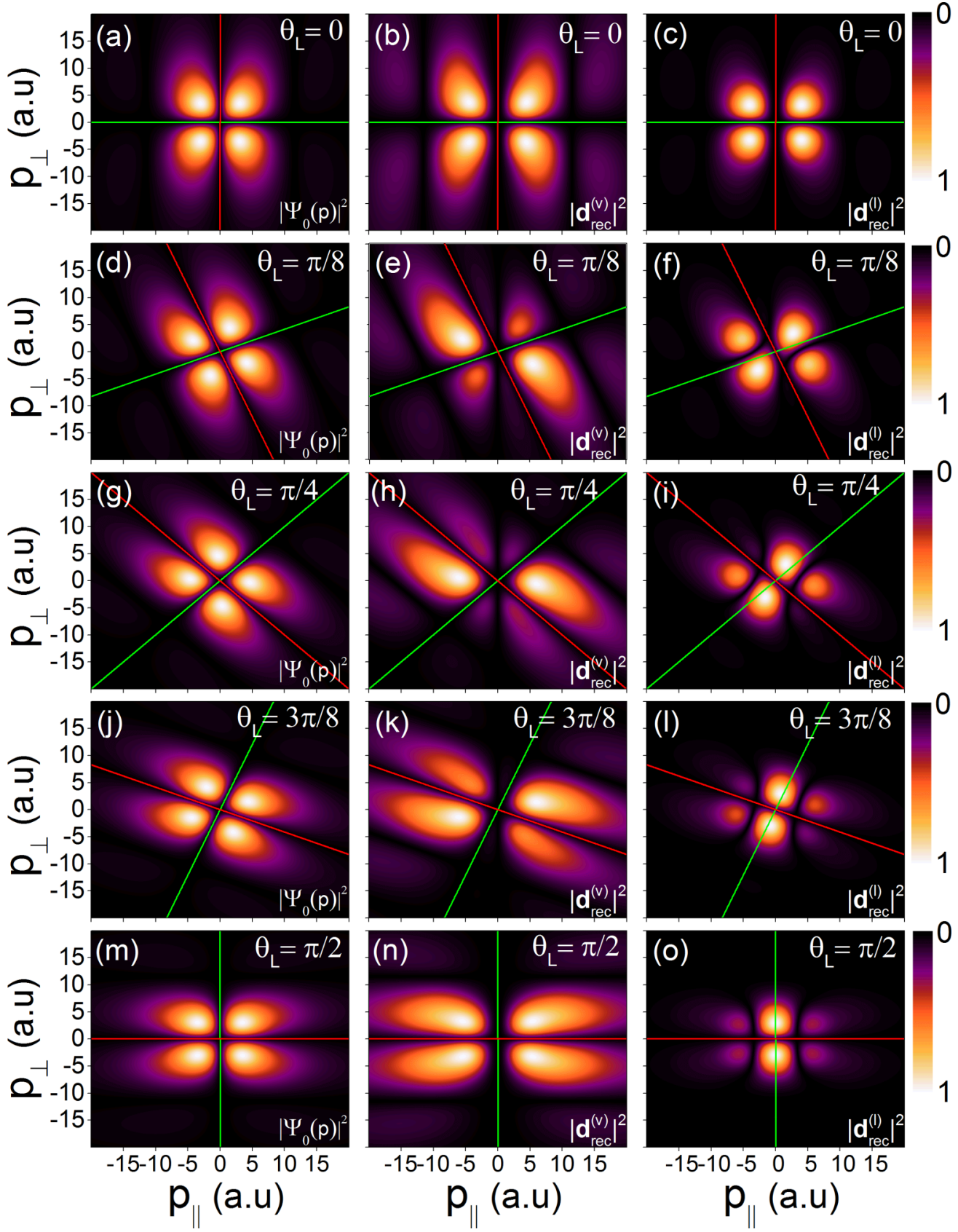

FIG. 5. In the first, second, and third columns we compare the probability density $\left|\Psi_{0}(\mathbf{p})\right|^{2}$ in momentum space with the absolute squares of the dipole matrix elements $d_{\text {rec }}^{(v)}\left(\mathbf{p} \cdot \hat{\epsilon}_{\|}\right)$and $d_{\text {rec }}^{(l)}\left(\mathbf{p} \cdot \hat{\epsilon}_{\|}\right)$along the major polarization axis, respectively, for the $\mathrm{HOMO}$ of $\mathrm{O}_{2}$. The alignment angle $\theta_{L}$ is increased from the top row $\theta_{L}=0$ to the bottom row $\theta_{L}=\pi / 2$ in increments of $\delta \theta_{L}=\pi / 8$. The $\mathrm{HOMO}$ of $\mathrm{O}_{2}$ is a $1 \pi_{g}$ orbital where $I_{p}=12 \mathrm{eV}$ and the internuclear separation is $R=2.28 \mathrm{a}$.u. The green and red lines in all the panels indicate the orientation of nodal planes constructed using atomic basis functions at single and different atomic centers, respectively. The quantity in each panel has been normalized by its maximum value.

These distortions are related to artifacts in the recombination dipole matrix elements, which leads to geometrical features that do not exist in the HOMO. In the present framework, the nodal structures are constructed in two ways. One may either employ nodes in the atomic orbitals at a single center in the molecule, or the sum or subtraction of atomic orbitals at different centers within the LCAO approximation. The former type of construction causes the suppressions at $\theta_{L}=n \pi$, while the latter lead to the suppressions at $\theta_{L}=(2 n+1) \pi / 2$. The velocity form of the SFA along the major polarization axis multiplies the momentum-space wave function by $p_{\|}$, while the length form of the SFA takes the partial derivative $\partial_{p_{\|}} \psi_{a}(\mathbf{p})$ of the atomic momentum wave functions used to construct the orbital [see Eq. (9)]. Both procedures modify the nodal structures constructed using a single center. This spurious behavior becomes evident as the molecule rotates.

This is exemplified in Fig. 5, where we display the HOMO probability density $\left|\Psi_{0}(\mathbf{p})\right|^{2}$ for $\mathrm{O}_{2}$ in momentum space, to- 
gether with the absolute squares of the dipole matrix elements $d_{\mathrm{rec}}^{(v)}\left(\mathbf{p} \cdot \hat{\epsilon}_{\|}\right)$and $d_{\mathrm{rec}}^{(l)}\left(\mathbf{p} \cdot \hat{\epsilon}_{\|}\right)$along the major polarization axis, for several alignment angles $\theta_{L}$. For $\theta_{L}=0$, the three pictures are similar, with four lobes separated by two orthogonal nodal planes [see first row in the figure]. For $\theta_{L} \neq 0$, however, this scenario changes, as shown in the remaining rows of the figure. While $\left|\Psi_{0}(\mathbf{p})\right|^{2}$ does not alter its structure and merely rotates, for the velocity form there is an additional nodal plane at $p_{\|}=0$. For the length form, the behavior is more extreme and the node constructed with a single center begins to warp, split, and shift away from the original shape and orientation of $\Psi_{0}(\mathbf{p})$ indicated by the green lines.

Both structures can be understood by inspecting the two prefactors. In the velocity form, $p_{\|} \psi_{a}(\mathbf{p})$ implies that there will be a suppression at $p_{\|}=0$. For $\theta_{L}=n \pi$, this condition coincides with the nodal plane given by $\tan \theta_{L}=p_{\perp} / p_{\|}$, which is obtained by imposing $\psi_{a}(\mathbf{p})=0$. For other angles, however, it leads to the spurious nodal structure. For the length form, the two terms in $\partial_{p_{\|}} \psi_{a}(\mathbf{p})$ lead, in general, to structures that are quadratic in $p_{\|}$. Specifically, Eq. (13) moves the suppression away from the axis $p_{\|}=0$, and the term given by Eq. (14) gives the above-mentioned warping. Once more, these spurious effects disappear for $\theta_{L}=n \pi$. These artifacts are overlooked by linearly polarized fields, as the electron's angle of return is always vanishing. This means that linearly polarized fields only probe the $p_{\|}$axis in Fig. 5. If the nodal planes are not parallel to this axis, the returning electron will "see" a nonvanishing probability density and no suppression will occur. Hence, linearly polarized fields can only probe the nodal planes at multiples of $\pi / 2$, for which the distortions cannot be seen along this axis.

The length or velocity form of the SFA dipole matrix elements has caused a lot of debate. In the single-active electron, single-active orbital approximation, it is known that the velocity form is superior in predicting structural interference minima, and provides the best agreement with the double-slit physical picture [39]. However, for linearly polarized driving fields the spurious terms introduced by the length form are well understood and easy to eliminate. They are caused by the lack of orthogonality between the bound and continuum states that exists in the SFA [33,37], and are absent from the start in the expressions used in this paper [see Eq. (9)].

The results in Figs. 3 to 5 tell us that, although for a linearly polarized field the form of the dipole operator may not make much difference, to the nodal suppressions in the HHG spectrum for an elliptically polarized field it does. This is because in this type of field the returning electron can probe dynamics of the wave function that would previously be unreachable. This exposes other artifacts in both forms of the SFA dipole, which are more difficult to eliminate. Nonetheless, the velocity form provides better results, if compared to the length form.

\section{Phase and field selections}

Our previous results show that the electron's angle of return modifies the suppressions in the HHG spectra near nodal planes. However, if one wishes to observe these shifts experimentally, they should be large and occur throughout the
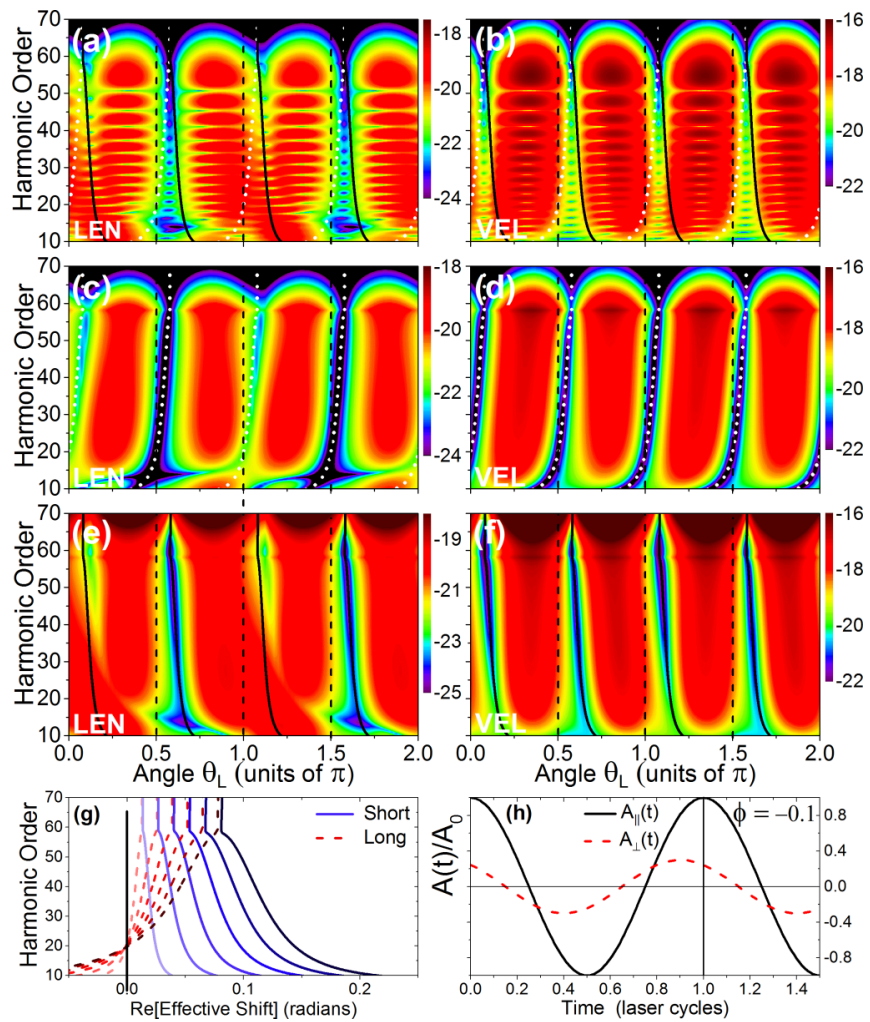

FIG. 6. In panels (a) to (f), we show $\left|M_{\|}(\Omega)\right|^{2}$ calculated using the length (first column) and the velocity (second column) forms of the SFA, plotted in a logarithmic scale. The first, second, and third rows have been calculated using the coherent superposition of the dominant orbits [panels (a) and (b)], and the individual contributions of the long [panels (c) and (d)] and short orbits [panels (e) and (f)], respectively. The parameters used are the same as in Fig. 2, but with a time delay $\phi=-0.1$ between the parallel and perpendicular waves. The black dashed lines indicate the positions of the nodal-plane suppressions for a linearly polarized field, while the white short dashed and solid black curves give the calculated positions of the suppressions for the long and short orbits, respectively, for elliptically polarized fields. In panel $(\mathrm{g})$ we have plotted the real parts of the effective shifts $\zeta\left(t, t^{\prime}\right)$ as functions of the harmonic order computed for the long (red dashed curves) and short (blue solid curves) orbits in laser fields of increasing ellipticity and the same relative phase, intensity, and frequency as in panels (a) to (f). The ellipticity is increased from $\xi=$ 0 to $\xi=0.3$ in increments of $\delta \xi=0.05$. A lighter color indicates a higher ellipticity and a vanishing shift is indicated by a vertical black line. Panel (h) provides a schematic representation of the major and minor components of the vector potential $\mathbf{A}(\mathrm{t})$ for ellipticity $\xi=0.3$ and relative phase $\phi=-0.1$. The electron return time at $t=2 \pi / \omega$ is indicated by the thick vertical black line in the figure. For simplicity, all fields have been normalized to the vector potential amplitude $\mathrm{A}_{0}=\mathrm{E}_{0} / \omega$.

whole spectrum, or at least in a broad harmonic range. Below we explore some fields choices which are favorable to this behavior.

One can, for instance, choose a different time delay between both waves in order to guarantee that. An example is provided in Fig. 6, for which we have chosen a phase difference of $\phi=-0.1$, using the whole dominant pair, the long and the short orbits [first, second, and third rows, respectively]. For 
comparison, we include results in the length [Figs. 6(a), 6(c), and 6(e)] and velocity [Figs. 6(b), 6(d), and 6(f)] forms of the SFA. For the short orbit, the shifts are large and present for the whole harmonic range, including the cutoff region. Indeed, Fig. 6(g), in which $\operatorname{Re}\left[\zeta\left(t, t^{\prime}\right)\right]$ is plotted, shows a large positive shift for the short orbit, which remains throughout the spectra. For the long orbit the behavior is more complex, but in both cases the residual shift at the cutoff is large. This is due to the fact that $A_{\perp}(t)$ is nonvanishing and positive at the cutoff return times [see Fig. 6(h)]. Once more, we see a better overall agreement between the analytical condition and the velocity form of the SFA. For the coherent superposition of the two orbits [Figs. 6(a) and 6(b)], despite some blurring in the plateau and threshold harmonics, the shift can be seen very clearly at the cutoff. Only the velocity form gives the correct shifts [Fig. 6(b)].

Another option would be to consider two-color orthogonal fields, for which the electron has a higher probability of return. This is an advantage over elliptically polarized fields, for
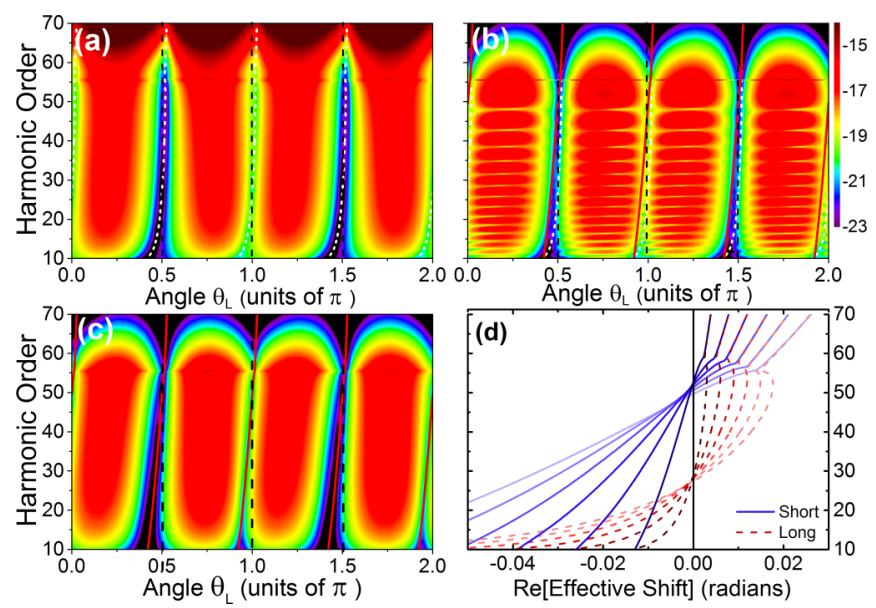

FIG. 7. In panels (a) to (c), we show the transition probabilities $\left|M_{\|}(\Omega)\right|^{2}$ along the major polarization axis plotted in a logarithmic scale as functions of the alignment angle $\theta_{L}$ for $\mathrm{O}_{2}$ (ionization potential $I_{p}=12 \mathrm{eV}$ and internuclear separation $R=2.28$ a.u.). The parameters used are the same as in Fig. 2, but with $n=2$ for the perpendicular wave, which is in phase $(\phi=0)$ with the parallel component of the laser field. Panels (a) and (c) give the individual contributions from the long and short orbits, respectively, while (b) shows the result using their coherent superposition. The black dashed lines indicate the positions of the nodal-plane suppressions in the spectrum for a linearly polarized field, while the white short dashed and red curves give the calculated positions of the suppressions for the long and short orbits, respectively, for elliptically polarized fields. The increase in the harmonic yields after the cutoff observed in panel (a) is related to a breakdown of the standard saddle-point approximation which occurs to the long orbits for this particular phase difference (for details, see Ref. [44]). In panel (d) we have plotted the real parts of the effective shifts $\zeta\left(t, t^{\prime}\right)$ as functions of the harmonic order computed for the long (red dashed curves) and short (solid blue curves) orbits in laser fields of increasing ellipticity and the same relative phase, intensity, and frequency as in panels (a), (b), and (c). The ellipticity is increased from $\xi=0$ to $\xi=0.3$ in increments of $\delta \xi=0.05$. A lighter color indicates a higher ellipticity and a vanishing shift is indicated by a vertical black line. The color map on the upper right column refers to panels (a) to (c). which it decreases as $\xi$ increases, eventually leading to low HHG efficiency. In Fig. 7, we present transition probabilities computed in a logarithmic scale for two-color fields and relative phase $\phi=0$. We consider a coherent superposition of the two dominant orbits [Fig. 7(b)], together with the individual contributions from the long and short orbits [Figs. 7(a) and 7(c), respectively]. The figure shows that the shifts in the nodal-plane suppressions are much smaller than those obtained in the elliptically polarized case. For instance, for $\xi=$ 0.3 , an elliptical field may lead to shifts up to $\operatorname{Re}\left[\zeta\left(t, t^{\prime}\right)\right]=$ 0.25 , while for a two-color field the effective shift reaches up to $\operatorname{Re}\left[\zeta\left(t, t^{\prime}\right)\right]=0.05$. This can be seen by comparing the effective shifts in Fig. 7(d) with the elliptical-field examples in Figs. 3(c) and 6(g). Hence, a two-color field would be less suitable for finding the shift in experiments, despite the higher probability of return.

\section{CONCLUSIONS}

In conclusion, this paper shows that nodal planes can be used as a tool in order to infer an electron's angle of return from high-order-harmonic spectra in molecules. This may provide an additional resource to steer electron motion in the continuum and probe systems which are difficult to access otherwise. Signatures of this angle are imprinted in the spectra as distortions in the nodal-plane suppressions. While for linearly polarized fields these suppressions are well known and occur at fixed alignment angles, if the fields are orthogonally polarized they are orbit and harmonic dependent. They can be controlled by changing the driving-field parameters, such as the relative phase, intensity, and frequency ratios between the two orthogonal waves. Nodal planes have advantages in comparison with two-center interference, which was addressed in our previous publications [16,19]. First, the shifts are in general larger, and spread across a broader harmonic region. Second, the distortions caused by the angle of return near nodal planes are in principle easier to identify as the suppressions are stronger. This would facilitate their observation in a realistic setting.

On a more technical note, we have also verified that the analytic expression for this angle, which was derived in [16], also works around nodal planes. This actually confirms that the shift encountered in [16] can be interpreted as a dynamical effect which is not exclusive to two-center interference. The suppressions encountered also expose limitations in the strongfield approximations, which have never been found for linearly polarized fields, or for two-center interference. They consist of artifacts in the recombination dipole matrix element, which, within our framework, gives the HHG suppressions. They occur both for the length and the velocity form of the dipole operator. Nonetheless, while for the velocity form they lead to a light blurring around the analytical condition, in the length form they are very extreme and lead to exaggerated distortions. Hence, the length form of the SFA should be avoided when mapping nodal planes using orthogonal fields.

Because the shifts are orbit dependent, they may be difficult to extract unless a particular return event can be singled out. This problem can, however, be solved through propagation, as the two dominant orbits phase match differently [48,49], aided by polarization gating [50]. Examples of this trajectory 
selection have been provided in our previous publication [19]. Another feature which will lead to a suppression of the contributions from the longer orbits already at the singlemolecule level is bound-state depletion. Depletion has been neglected in this work, and will gain in relevance as the driving-field intensity increases.

We have also found that elliptically polarized fields provide better conditions for observing the shifts experimentally, in comparison with OTC fields. First, using an elliptically polarized field gives rise to a much larger shift. Second, for these fields the shift remains the same for subsequent half cycles, while for OTC fields it flips [16]. This was a major problem in [19], which was overcome by using a few-cycle pulse in which a specific cycle was dominant in the region of interest. For elliptical fields, however, there is no need to restrict ionization events to a single half cycle. Hence, any pulse length can be employed.

Finally, in this work we have considered (a) the strongfield approximation and the steepest descent method and (b) the single-active electron, single-active orbital approximation. Hence, one must guarantee that approximations (a) and (b) hold in the parameter range of interest.

The strong-field approximation neglects the binding potential when the electron is in the continuum. This potential will alter the topology of the electron orbits, which may be important when dealing with coherent superpositions (for examples in strong-field ionization, see [51-53]). Furthermore, Coulomb focusing will introduce extra phases [54] and is expected to alter the electron's angle of return. The steepest descent method eliminates the momentum spread associated with the electronic wave packet [55] and also leads to an over-enhancement of the long orbit [56,57]. Still, on a qualitative level, there is evidence that the angle of return may be estimated by the SFA. This evidence has been provided by comparisons with experimental data [36] or with classical-trajectory methods which incorporate the initial electron momentum spread and the binding potential [4].

The single-active electron, single-active orbital approximation requires that the influence of the inner orbitals and of the core dynamics be minimized in the parameter range of interest. If the driving field intensity is such that the HOMO does not become strongly depleted, and if structural effects prevail over dynamical features, the method discussed here is reliable. The latter features have been found to be dominant in the cutoff region, or near the two-center interference minimum. The present work has been performed in the plateau and far away from the two-center minimum, so that we expect structural effects to be dominant $[58,59]$.

\section{ACKNOWLEDGMENTS}

This work has been funded by the UK Engineering and Physical Sciences Research Council (EPSRC) (Grant No. EP/J019240/1 and doctoral training prize) and by UCL (Impact Studentship). We thank M. Kitzler and B. B. Augstein for useful discussions.
[1] N. Dudovich, O. Smirnova, J. Levesque, Y. Mairesse, M. Yu Ivanov, D. M. Villeneuve, and P. B. Corkum, Nat. Phys. 2, 781 (2006).

[2] M. Kitzler, X. Xie, A. Scrinzi, and A. Baltuska, Phys. Rev. A 76, 011801(R) (2007).

[3] D. Shafir, Y. Mairesse, D. M. Villeneuve, P. B. Corkum, and N. Dudovich, Nat. Phys. 5, 412 (2009).

[4] M. Kitzler and M. Lezius, Phys. Rev. Lett. 95, 253001 (2005).

[5] H. Niikura, N. Dudovich, D. M. Villeneuve, and P. B. Corkum, Phys. Rev. Lett. 105, 053003 (2010).

[6] H. Niikura, H. J. Wörner, D. M. Villeneuve, and P. B. Corkum, Phys. Rev. Lett. 107, 093004 (2011).

[7] H. Yun, K.-M. Lee, J. H. Sung, K. T. Kim, H. T. Kim, and C. H. Nam, Phys. Rev. Lett. 114, 153901 (2015).

[8] P. B. Corkum, Phys. Rev. Lett. 71, 1994 (1993).

[9] M. Kitzler, X. Xie, S. Roither, A. Scrinzi, and A. Baltuska, New J. Phys. 10, 025029 (2008).

[10] M. V. Frolov, N. L. Manakov, T. S. Sarantseva, A. A. Silaev, N. V. Vvedenskii, and A. F. Starace, Phys. Rev. A 93, 023430 (2016).

[11] D. B. Milošević and W. Becker, Phys. Rev. A 66, 063417 (2002).

[12] M. Lein, N. Hay, R. Velotta, J. P. Marangos, and P. L. Knight, Phys. Rev. A 66, 023805 (2002).

[13] M. Lein, N. Hay, R. Velotta, J. P. Marangos, and P. L. Knight, Phys. Rev. Lett. 88, 183903 (2002).

[14] M. Lein, J. Phys. B 40, R135 (2007).

[15] B. Augstein and C. F. de Morisson Faria, Mod. Phys. Lett. B 26, 1130002 (2012).

[16] T. Das, B. B. Augstein, and C. Figueira de Morisson Faria, Phys. Rev. A 88, 023404 (2013).
[17] S. Odžak and D. B. Milošević, Phys. Rev. A 82, 023412 (2010).

[18] S. Odžak and D. Milošević, J. Phys. B 44, 125602 (2011).

[19] T. Das, B. B. Augstein, C. Figueira de Morisson Faria, L. E. Chipperfield, D. J. Hoffmann, and J. P. Marangos, Phys. Rev. A 92, 023406 (2015).

[20] S. Odžak and D. B. Milošević, Phys. Rev. A 79, 023414 (2009).

[21] B. B. Augstein and C. Figueira de Morisson Faria, J. Mod. Opt. 58, 1173 (2011).

[22] B. K. McFarland, J. P. Farrell, P. H. Bucksbaum, and M. Gühr, Science 322, 1232 (2008).

[23] O. Smirnova, Y. Mairesse, S. Patchkovskii, N. Dudovich, D. Villeneuve, P. Corkum, and M. Y. Ivanov, Nature (London) 460, 972 (2009).

[24] D. Pavičič, K. F. Lee, D. M. Rayner, P. B. Corkum, and D. M. Villeneuve, Phys. Rev. Lett. 98, 243001 (2007).

[25] M. Abu-samha and L. B. Madsen, Phys. Rev. A 80, 023401 (2009).

[26] S. Petretti, Y. V. Vanne, A. Saenz, A. Castro, and P. Decleva, Phys. Rev. Lett. 104, 223001 (2010).

[27] V. R. Bhardwaj, D. M. Rayner, D. M. Villeneuve, and P. B. Corkum, Phys. Rev. Lett. 87, 253003 (2001).

[28] M. Lein, J. Phys. B 36, L155 (2003).

[29] R. Kopold, W. Becker, and M. Kleber, Phys. Rev. A 58, 4022 (1998).

[30] C. B. Madsen and L. B. Madsen, Phys. Rev. A 74, 023403 (2006)

[31] C. B. Madsen and L. B. Madsen, Phys. Rev. A 76, 043419 (2007).

[32] C. C. Chirilă and M. Lein, Phys. Rev. A 73, 023410 (2006).

[33] C. Figueira de Morisson Faria, Phys. Rev. A 76, 043407 (2007). 
[34] C. Figueira de Morisson Faria and B. B. Augstein, Phys. Rev. A 81, 043409 (2010).

[35] A. Etches and L. B. Madsen, J. Phys. B 43, 155602 (2010).

[36] C. Hutchison, S. Houver, N. Lin, D. J. Hoffmann, F. McGrath, T. Siegel, D. R. Austin, A. Zar, P. Salieres, and J. P. Marangos, J. Mod. Opt. 61, 608 (2014).

[37] O. Smirnova, M. Spanner, and M. Ivanov, J. Mod. Opt. 54, 1019 (2007).

[38] C. Granados and L. Plaja, Phys. Rev. A 85, 053403 (2012).

[39] C. C. Chirilă and M. Lein, J. Mod. Opt. 54, 1039 (2007).

[40] E. V. van der Zwan, C. C. Chirilă, and M. Lein, Phys. Rev. A 78, 033410 (2008).

[41] E. Hijano, C. Serrat, G. N. Gibson, and J. Biegert, Phys. Rev. A 81, 041401(R) (2010).

[42] X. Zhu, M. Qin, Y. Li, Q. Zhang, Z. Xu, and P. Lu, Phys. Rev. A 87, 045402 (2013).

[43] M. Lewenstein, Ph. Balcou, M. Yu. Ivanov, A. L'Huillier, and P. B. Corkum, Phys. Rev. A 49, 2117 (1994).

[44] C. Figueira de Morisson Faria, H. Schomerus, and W. Becker, Phys. Rev. A 66, 043413 (2002).

[45] O. Smirnova, S. Patchkovskii, Y. Mairesse, N. Dudovich, D. Villeneuve, P. Corkum, and M. Yu. Ivanov, Phys. Rev. Lett. 102, 063601 (2009).

[46] M. F. Guest, I. J. Bush, H. J. J. Van Dam, P. Sherwood, J. M. H. Thomas, J. H. Van Lenthe, R. W. A. Havenith, and J. Kendrick, Mol. Phys. 103, 719 (2005).
[47] P. Antoine, A. L'Huillier, and M. Lewenstein, Phys. Rev. Lett. 77, 1234 (1996).

[48] Ph. Balcou, P. Salières, A. L'Huillier, and M. Lewenstein, Phys. Rev. A 55, 3204 (1997).

[49] M. B. Gaarde, J. L. Tate, and K. J. Schafer, J. Phys. B 41, 132001 (2008).

[50] D. J. Hoffmann, C. Hutchison, A. Zaïr, and J. P. Marangos, Phys. Rev. A 89, 023423 (2014).

[51] T. M. Yan and D. Bauer, Phys. Rev. A 86, 053403 (2012).

[52] T. M. Yan, S. V. Popruzhenko, M. J. J. Vrakking, and D. Bauer, Phys. Rev. Lett. 105, 253002 (2010).

[53] X.-Y. Lai, C. Poli, H. Schomerus, and C. Figueira de Morisson Faria, Phys. Rev. A 92, 043407 (2015).

[54] Lisa Torlina, Jivesh Kaushal, and Olga Smirnova, Phys. Rev. A 88, 053403 (2013).

[55] C. C. Chirilă and M. Lein, Phys. Rev. A 80, 013405 (2009).

[56] M. B. Gaarde and K. J. Schafer, Phys. Rev. A 65, 031406(R) (2002).

[57] J. A. Pérez-Hernandez, J. Ramos, L. Roso, and L. Plaja, Laser Phys. 20, 1044 (2010).

[58] K. Kato, S. Minemoto, and H. Sakai, Phys. Rev. A 84, 021403(R) (2011).

[59] A. Rupenyan, P. M. Kraus, J. Schneider, and H. J. Wörner, Phys. Rev. A 87, 033409 (2013). 